\title{
Geometric rules for terrestrial radionavigation multipath mitigation by averaging
}

\author{
Nicolas Schneckenburger, Sherman Lo, Michael Walter, Uwe-Carsten Fiebig
}

\begin{abstract}
In this contribution, we present a method to analyze the effectiveness of multipath mitigation by extended averaging in the context of terrestrial radionavigation. We derive the requirements of extended averaging on the geometry between a ground station and an aircraft. Using that knowledge we are able to analyze the effectiveness of extended averaging for real world flight trajectories.
\end{abstract}

\section{INTRODUCTION}

In the future, pilots in civil aviation will mainly rely on global navigation satellite systems (GNSS) as their primary means of navigation. Nevertheless, terrestrial radionavigation systems will continue to play a vital role as alternative positioning navigation and timing (APNT) systems in the future navigation infrastructure [1]. APNT systems will provide the ability to continue operations, should the primary satellite based navigation infrastructure become unavailable. Currently, several proposals for terrestrial APNT radionavigation systems exist [2], [3], [4].

Multipath propagation has been identified as the major threat for the navigation performance in terrestrial APNT [5], [6], [7]. Signal components, reflected off buildings or the ground surrounding the ground station, interfere with the direct signal used for the range estimation. The resulting range estimation errors have been observed to exceed $150 \mathrm{~m}$ [8]. Errors introduced by multipath propagation have to be mitigated or detected, if the performance of current terrestrial radionavigation systems, e.g. the currently widely used distance measurement equipment (DME), are to be improved in order to support new more fuel-efficient flight procedures.

Receiver algorithms exploiting not only the envelope but also the phase of the received signal, have been identified as a method to mitigate the influence of multipath propagation on range estimation [5], [6].

A powerful multipath mitigation technique is to average over a long period of time - extended averaging (EA). This technique can be aided with additional information, such as carrier phase, and this is termed carrier smoothing. The method developed is applicable to any algorithm improving range estimation using averages of the signal phase or envelope measurements.

So far the applicability of EA with respect to the typical geometry between aircraft and a terrestrial radionavigation system has not been evaluated. Therefore, in this publication we perform a theoretical evaluation, under which circumstances EA can be applied to mitigate errors caused by multipath propagation.

Throughout the paper, the following notation is used: Scalars are denoted by light lowercase, vectors by bold lowercase and matrices bold uppercase letters. $\|\cdot\|$ denotes the absolute value of a scalar or Euclidean norm of a vector and $\tan _{2}^{-1}(\cdot, \cdot)$ the four-quadrant inverse tangent. $c_{\mathrm{a}}$ denotes the speed of light in air.

\section{EXtended AVERAGing AND ITS REQUIREMENTS ON THE GEOMETRY}

In the following section, we will show how the aircraft location and speed vector relative to the ground station can influence the effectiveness of EA. 


\section{A. Multipath propagation and range estimation error}

The range or pseudorange estimation error of a terrestrial aeronautical navigation system mainly depends on the propagation characteristics of the radio channel.

Range estimation suffers if the signal received via the direct line-of-sight (LoS) propagation path overlaps with a multipath component (MPC) received via a reflection off the ground or a surrounding building. The resulting error for a specific system can be analyzed using a multipath error envelope.

The range estimation error depends on the amplitude of the complex propagation path weight $\left\|\alpha_{\mathrm{M}}\right\|$ and delay $\tau_{\mathrm{M}}$ of the MPC, relative to the amplitude $\left\|\alpha_{\mathrm{L}}\right\|$ and delay $\tau_{\mathrm{L}}$ of the LoS path. For most systems, the range estimation error scales with an increasing relative amplitude of a MPC $\left\|\alpha_{\mathrm{M}, \text { rel }}\right\|=\left\|\alpha_{\mathrm{M}}\right\| /\left\|\alpha_{\mathrm{L}}\right\|$. In this publication we only focus on the case where the MPC receive power is smaller than the LoS received power. Without that constraint, the range estimation error caused by a MPC is theoretically unlimited.

The dependency of the range estimation error on the relative delay of a MPC, $\tau_{\mathrm{M} \text {,rel }}=\tau_{\mathrm{M}}-\tau_{\mathrm{L}}$ strongly differs among employed systems and their associated bandwidth $f_{\mathrm{BW}}$. However, navigation systems are usually vulnerable to delays below their inverse bandwidth $f_{\mathrm{BW}}$; i.e. $\tau_{\mathrm{M}}<1 / f_{\mathrm{BW}}$, e.g. the critical relative delays $\tau_{\mathrm{M}, \mathrm{c}}$ for DME are between $0.3 \mu \mathrm{s}$ and $2.3 \mu \mathrm{s}$ [5].

If a MPC is received with a smaller power than the LoS path, i.e. $\left\|\alpha_{\mathrm{M} \text {,rel }}\right\|<1$, its influence on the range estimation can be mitigated using EA. EA considers the evolution of the estimated delay over time. If the MPC delay $\tau_{\mathrm{M}}$ changes at a different rate than the LoS delay $\tau_{\mathrm{L}}$, the MPC induced range estimation error can be smoothed out. The rate of change of the carrier phase of a propagation path is expressed by its Doppler frequency $f$. By applying EA, we assume the error caused by a MPC with a relative Doppler frequency $f_{\mathrm{M} \text {,rel }}$ can be mitigated if the averaging time is chosen as $T_{\mathrm{av}}>f_{\mathrm{M}, \mathrm{rel}}^{-1}$. Therefore, given an averaging time of $T_{\mathrm{av}}=10 \mathrm{~s}$, the critical value for the relative Doppler frequency $f_{\mathrm{M}, \mathrm{c}}$ is $-0.1 \mathrm{~Hz}<f_{\mathrm{M}, \mathrm{c}}<0.1 \mathrm{~Hz}$.

\section{B. Reflector locations of constant delay and Doppler frequency}

Given the transmit antenna location $\boldsymbol{d}_{\mathrm{GS}}$, aircraft position $\boldsymbol{p}$ and movement vector $\boldsymbol{v}$, the locations of reflectors causing MPCs with a constant relative delay $\tau_{\mathrm{M}, \text { rel }}$ and Doppler frequency $f_{\mathrm{M}, \text { rel }}$ can be calculated. All coordinates are given in a local east-north-up (ENU) coordinate system centered at the ground station location, i.e. $\boldsymbol{d}_{\mathrm{GS}}=[0,0,0]^{\mathrm{T}}$. In the following, we assume single bounce reflections. Hereby, we expect all MPCs with an amplitude capable of significantly degrading the ranging performance to be covered. In measurements also reflections with two bounces have been observed to have significant relative amplitude $\left\|\alpha_{\mathrm{M}}\right\|$. However, in that case one of the reflection angles usually approaches $90^{\circ}$, leading to an almost identical geometry as for a single bounce reflection.

Points with constant delay lie on an ellipsoid with the transmit and receive antenna as focal points as depicted in Fig. 1.

Using the position vector $\boldsymbol{d}=\left[d_{\mathrm{E}}, d_{\mathrm{N}}, d_{\mathrm{U}}\right]^{\mathrm{T}}$, the ellipsoid defined by the absolute delays of the LoS and $\mathrm{MPC}, \tau_{\mathrm{L}}$ and $\tau_{\mathrm{M}}$ respectively, is given by

$$
\left(\underline{\boldsymbol{R}}_{\tau, U} \underline{\boldsymbol{R}}_{\tau, N}\left(\boldsymbol{d}-\boldsymbol{d}_{\tau}\right)\right)^{\mathrm{T}}\left[\begin{array}{ccc}
\frac{1}{a_{\tau}^{2}} & 0 & 0 \\
0 & \frac{1}{b_{\tau}^{2}} & 0 \\
0 & 0 & \frac{1}{b_{\tau}^{2}}
\end{array}\right] \underline{\boldsymbol{R}}_{\tau, U} \underline{\boldsymbol{R}}_{\tau, N}\left(\boldsymbol{d}-\boldsymbol{d}_{\tau}\right)=1
$$

The ellipsoid parameters are calculated as $a_{\tau}=\frac{\tau_{\mathrm{M}}}{2} c_{\mathrm{a}}, b_{\tau}=\sqrt{\left(\frac{\tau_{\mathrm{M}}}{2}\right)^{2}-\left(\frac{\tau_{\mathrm{L}}}{2}\right)^{2}}$ and $\boldsymbol{d}_{\tau}=\frac{p}{2}$. Hereby, $\underline{\boldsymbol{R}}_{\tau, N}$ and $\underline{\boldsymbol{R}}_{\tau, U}$ define the rotation matrices for the rotation around the $N$ and $U$ axis by the angles 


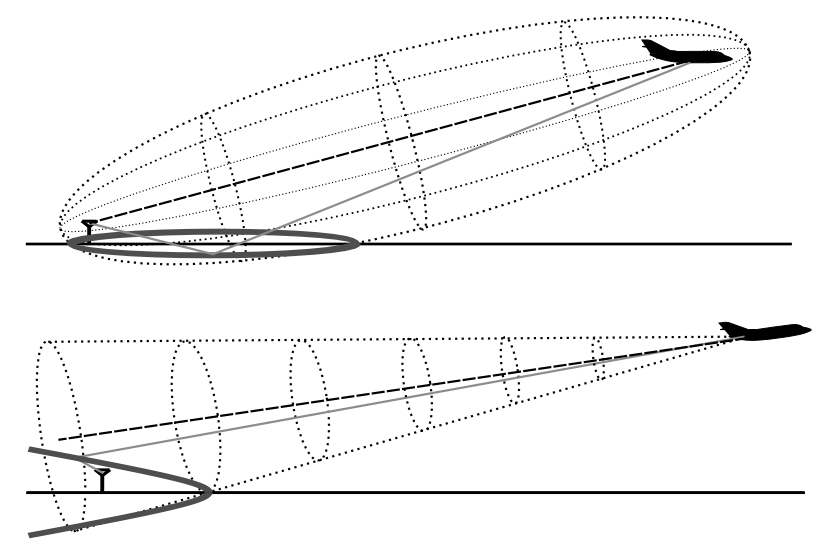

Fig. 1. Illustration of the shapes defined by reflectors with constant delay and Doppler frequency.

$\alpha_{\tau, N}=\tan _{2}^{-1}\left(p_{\mathrm{E}}, p_{\mathrm{N}}\right)$ and $\alpha_{\tau, U}=\tan _{2}^{-1}\left(\sqrt{p_{\mathrm{E}}^{2}+p_{\mathrm{N}}^{2}}, p_{\mathrm{N}}\right)$. Intersecting the ellipsoid with the ground plane results in an ellipse.

Similarly, points with a constant Doppler frequency lie on a cone centered on the aircraft position $\boldsymbol{p}$ and opening in the opposite direction of the aircraft movement vector $\boldsymbol{v}$ as illustrated in Fig. 1. The rotated cone, defined by the Doppler frequency of the $\mathrm{MPC} f_{\mathrm{M}}$, is given by

$$
\left(\underline{\boldsymbol{R}}_{f, U} \underline{\boldsymbol{R}}_{f, N}(\boldsymbol{d}-\boldsymbol{p})\right)^{\mathrm{T}}\left[\begin{array}{ccc}
\frac{1}{h_{f}^{2}} & 0 & 0 \\
0 & \frac{1}{h_{f}^{2}} & 0 \\
0 & 0 & 1
\end{array}\right] \underline{\boldsymbol{R}}_{f, U} \underline{\boldsymbol{R}}_{f, N}(\boldsymbol{d}-\boldsymbol{p})=0
$$

The cone parameter is calculated as $h_{f}=\tan \left(\cos ^{-1} \frac{f_{\mathrm{M}} c_{\mathrm{a}}}{|\boldsymbol{v}| f_{\mathrm{c}}}\right)$ with $f_{\mathrm{c}}$ the carrier frequency of the signal. The rotation angles for $\alpha_{\tau, N}$ and $\alpha_{\tau, U}$ are calculated as $\alpha_{f, N}=\tan _{2}^{-1}\left(v_{\mathrm{E}}, v_{\mathrm{N}}\right)$ and $\alpha_{f, U}=$ $\tan _{2}^{-1}\left(\sqrt{v_{\mathrm{E}}^{2}+v_{\mathrm{N}}^{2}}, v_{\mathrm{U}}\right)$. The intersection of the cone defined by a constant Doppler frequency with the ground plane results in either a hyperbola or an ellipse.

In both cases, assuming a ground station antenna height of $h_{\mathrm{GS}}$, reflector positions of constant delay $\tau_{\mathrm{M}}$ or Doppler frequency $f_{\mathrm{M}}$ can be found by setting $d_{\mathrm{U}}=-h_{\mathrm{GS}}$ in Eq. (1) and Eq. (2). The closed form solution used for calculation of presented results can be found by bringing Eq. (1) and Eq. (2) into the general scalar form of a quadric curve [9]

$$
Q_{1} x^{2}+2 Q_{2} x y+Q_{3} y^{2}+Q_{4} x+Q_{5} y+Q_{6}=0
$$

and then solving for $y=f(x)$. Although not mathematically complex, the calculation of the coefficients $Q_{1}$ through $Q_{6}$ is tedious and results in very long expressions.

\section{Critical zone for reflectors}

We define the critical zone as the area where a reflector causing a MPC with a critical relative delay $\tau_{\mathrm{M}, \mathrm{c}}$ and Doppler frequency $f_{\mathrm{M}, \mathrm{c}}$ can lie. As an example we assume a critical relative delay $0.3 \mu \mathrm{s}<$ $\tau_{\mathrm{M}, \mathrm{c}}<2.3 \mu \mathrm{s}$, values representative for the currently most commonly used terrestrial radionavigation system DME [5]. Choosing an averaging time of $T_{\mathrm{av}}=20 \mathrm{~s}$ leads to a critical Doppler frequency $-0.05 \mathrm{~Hz}<f_{\mathrm{M}, \mathrm{c}}<0.05 \mathrm{~Hz}$.

In Fig. 2 an example for such a critical zone is shown. The aircraft altitude is $h_{\mathrm{AC}}=5 \mathrm{~km}$ 

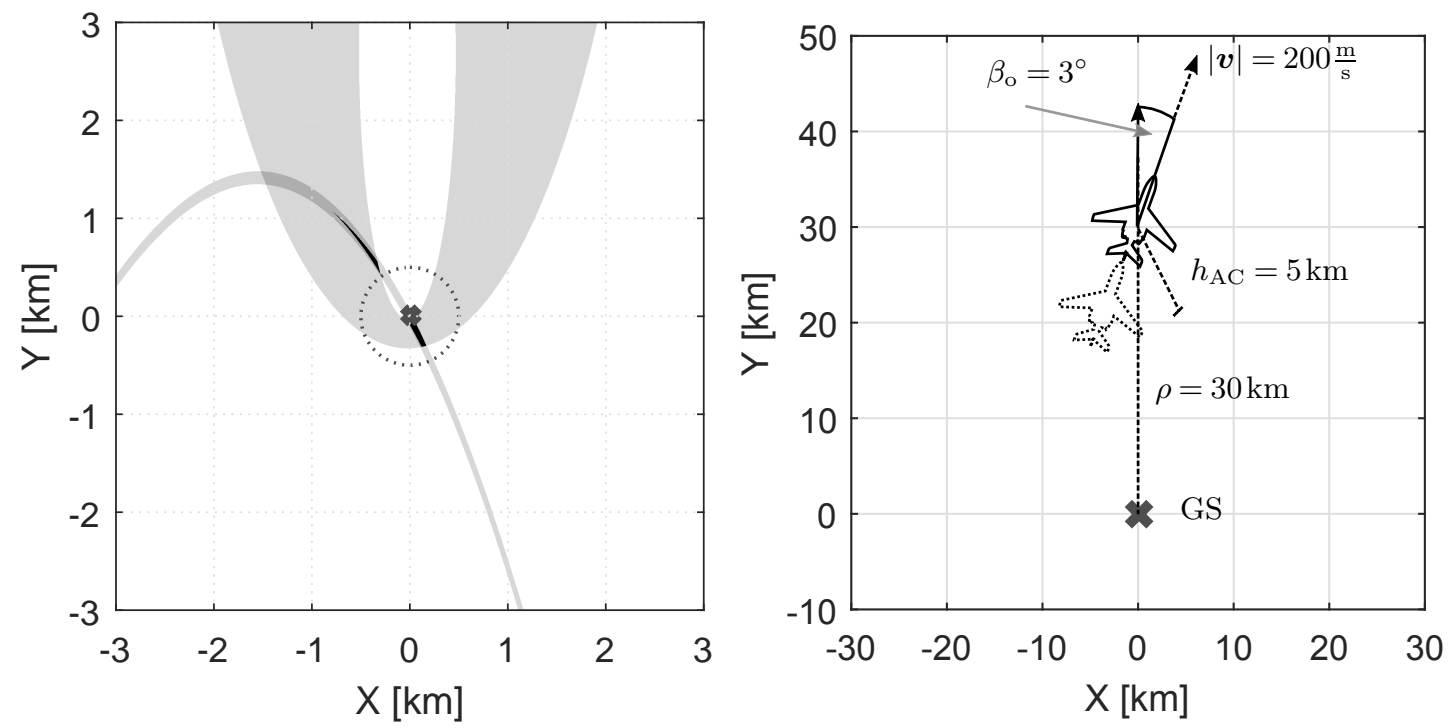

Fig. 2. Example for the critical zone for reflectors (left) for the illustrated scenario (right).

above ground level (AGL) and its distance to the ground station located at $[0,0]$, i.e. the range is $\rho=30 \mathrm{~km}$. The aircraft is flying at an absolute speed of $|\boldsymbol{v}|=200 \mathrm{~m} / \mathrm{s}$. Its movement direction (velocity) relative to the line between the aircraft and ground station, hereafter referred to as its ground station offset angle or short offset angle, is $\beta_{\mathrm{o}}=3^{\circ}$. The ground station antenna height is $h_{\mathrm{GS}}=5 \mathrm{~m}$. The dashed line bounds an area to be left clear in order to decrease the occurrence of critical MPCs (see Sec. III).

Fig. 2 shows the ellipses defined by the critical delays $\tau_{\mathrm{M}, \mathrm{c}}$ and hyperbolas defined by the critical Doppler frequencies $f_{\mathrm{M}, \mathrm{c}}$ in grayscale for one aircraft location. The black area denotes the intersection of critical zones if the aircraft is flying for $20 \mathrm{~s}$. A reflector located in the critical zone, e.g. a building, would cause a MPC with the potential to introduce large ranging errors which cannot be mitigated by EA.

\section{Critical zone for a moving aircraft}

The gray scale plot in Fig. 2 shows the critical zone for a single snapshot. However, as the aircraft moves, location and shape of the areas defined by the critical relative delay $\tau_{\mathrm{M}, \mathrm{c}}$ and Doppler frequency $f_{\mathrm{M}, \mathrm{c}}$ change. Thus, a reflector located in the critical zone at one snapshot may not lie in the critical zone one second later. For a MPC to have a strong negative effect on the range estimation, we assume that it has to maintain a critical relative delay over the full duration of the averaging time.

In Fig. 2, we mark in black the intersection of critical zones for an aircraft flying with the offset angle $\beta_{\mathrm{o}}=3^{\circ}$ for $20 \mathrm{~s}$. Due to the movement, the shapes defined by the critical delay $\tau_{\mathrm{M}, \mathrm{c}}$ and Doppler frequency $f_{\mathrm{M}, \mathrm{c}}$ change slightly from snapshot to snapshot. Thus, the remaining critical zone, the intersection of the critical zones for each snapshot, marked in black is smaller than the critical zone of single snapshot.

\section{ANALYSis of THE EFFECTIVENESS OF EXTENDED AVERAGING}

In the following section we use the method presented in Sec. II to calculate quantitative results about the geometry in which EA is most efficient. 


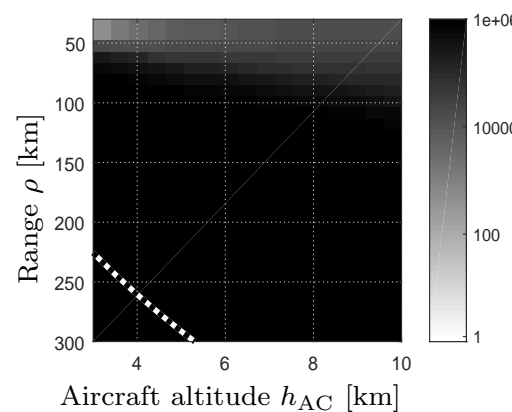

(a) $\beta_{\mathrm{o}}=0^{\circ}$

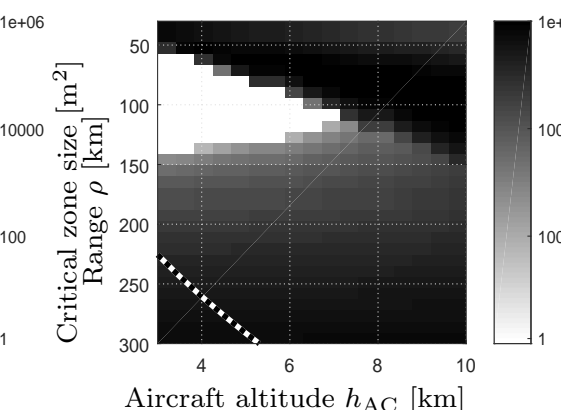

(b) $\beta_{\mathrm{o}}=2^{\circ}$

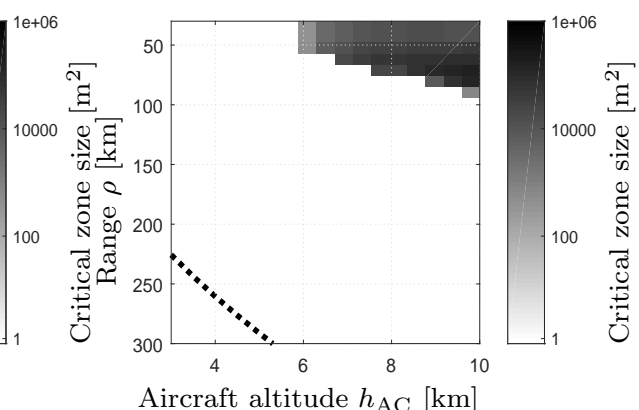

(c) $\beta_{\mathrm{o}}=5^{\circ}$

Fig. 3. Dependency of size of the critical zone on aircraft range $\rho$ and altitude $h_{\mathrm{AC}}$ for different offset angles $\beta_{\mathrm{o}}$. The dashed line marks the effective radio horizon.

TABLE I. Dependency of EA on offset angle $\beta_{\mathrm{o}}$ and $T_{\mathrm{av}}$.

\begin{tabular}{|l||c|c|c|c|c|}
\hline$T_{\mathrm{av}}[\mathrm{s}]$ & 5 & 10 & 20 & 30 & 60 \\
\hline$\beta_{\mathrm{o}, \min }\left[^{\circ}\right]$ & 47 & 15 & 8 & 5 & 3 \\
\hline
\end{tabular}

\section{A. Dependency of critical zone size on flight path}

When looking at the critical zone in a quantitative way, we observe the following: Independent of the aircraft flight parameters, such as range $\rho$, altitude $h_{\mathrm{AC}}$, offset angle $\beta_{\mathrm{o}}$, and speed $|\boldsymbol{v}|$, parts of the ground station's direct surroundings are usually in the critical zone. We assume that the navigation service provider (NSP) can maintain an area clear of possible reflectors, e.g. it is flat and covered by grass. A clear area, as indicated in Fig. 2, would greatly reduce the occurrence of critical MPCs.

Under the assumption above, the effectiveness of EA strongly depends on the offset angle $\beta_{\mathrm{o}}$ and averaging time $T_{\mathrm{av}}$. Fig. 3 shows the dependency of size of the remaining critical zone on aircraft range $\rho$ and altitude $h_{\mathrm{AC}}$ for different offset angles $\beta_{\mathrm{o}}$ using an averaging time of $T_{\mathrm{av}}=20 \mathrm{~s}$. Hereby, we assume that a circular area of radius $r_{\text {clear }}=500 \mathrm{~m}$ around the ground station is free of reflectors. For a small offset angle $\beta_{\mathrm{o}}$, the size of the remaining critical zone is usually very large. EA works best, if the ground station and the reflectors surrounding it are seen from different angles. The changing relative Doppler frequencies of the reflectors $f_{\mathrm{M} \text {,rel }}$ allows the mitigation of the introduced range estimation errors.

Tabular I lists the minimum offset angle $\beta_{\mathrm{o} \text {,min }}$ at which the remaining critical zone size is zero for different averaging times $T_{\mathrm{av}}$. Hereby, we limit the range to $20 \mathrm{~km}<\rho<300 \mathrm{~km}$ and altitude to $2 \mathrm{~km}<h_{\mathrm{AC}}<10 \mathrm{~km}$. We observe, that $\beta_{\mathrm{o} \text {,min }}$ increases for a decreasing $T_{\mathrm{av}}$. The aircraft traverses a smaller distance with a shorter averaging time $T_{\mathrm{av}}$. Therefore, the geometry changes, under which the ground station and reflectors are seen, are smaller and EA has reduced effect.

Similarly, decreasing the aircraft speed $|\boldsymbol{v}|$ has a negative effect on the effectiveness of EA. A smaller aircraft speed $|\boldsymbol{v}|$ leads to a smaller distance traveled during a constant averaging time $T_{\mathrm{av}}$.

\section{B. Application of the method to flight path design and testing}

In the following, we show an example how the method described above can be applied to evaluate or improve existing flight paths with respect to the applicability of EA. As an example we analyze the "Brigham City Three Arrival" to runway 16L at Salt Lake City International Airport (SLC). SLC was chosen as it represents a very challenging environment due the mountainous terrain surrounding it. Fig. 4 shows the arrival path and surrounding DME ground stations. Similarly to the path shown 


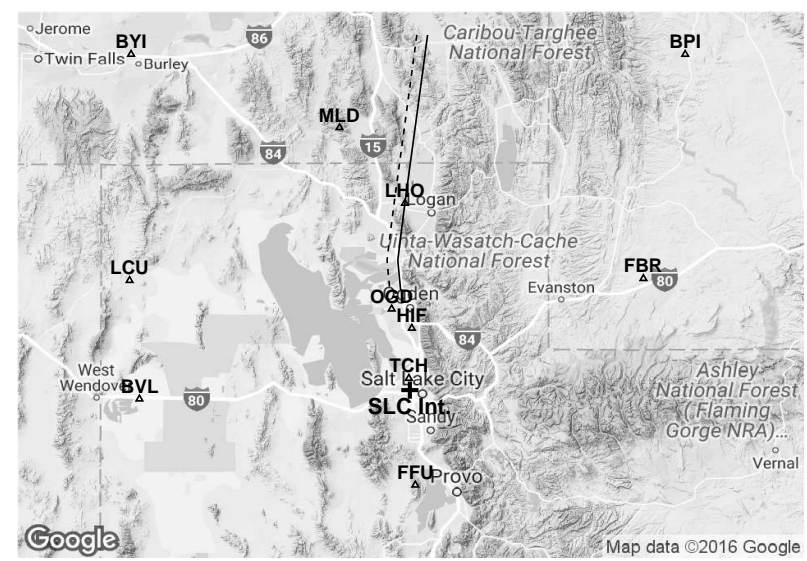

Fig. 4. "Brigham City Three Arrival" into SLC and surrounding DME stations. SLCs location is marked by a cross. (C) Google)

in Fig. 4, current flight paths are mainly designed in a way such that the pilot either flies directly towards or away from its currently designated ground station (LHO or OGD), i.e. $\beta_{\mathrm{o}}=0^{\circ}$.

Fig. 5 shows the availability of DME stations with a remaining critical zone size of zero. We assume a circular area of radius $r_{\text {clear }}=500 \mathrm{~m}$ around the ground station to be free of reflectors. To determine, if a station is visible at a certain time from the aircraft we employ the $3 \mathrm{D}$ coverage tool presented in [4]. The model takes into account shadowing by terrain as well areas with low received power due to nulls in the antenna patterns.

Comparing the different averaging times, $T_{\mathrm{av}}=5 \mathrm{~s}$ and $T_{\mathrm{av}}=20 \mathrm{~s}$, we observe that a longer averaging time decreases the requirements on the geometry. We observe from Fig. 5 that applying EA to the signals received from the two DME stations designated for the approach, LHO and OGD, may not always decrease errors introduced by multipath propagation. EA cannot be effective, as the aircraft is flying directly towards those stations.

One solution to this problem is to use different ground stations. Fig. 5 shows that, even in a challenging environment like the area surrounding SLC, enough suitable stations are visible. The geometry between most of those ground stations and the aircraft is well suited to apply EA.

The second solution is to slightly change the flight path in order to increase the offset angle relative to the two DME ground stations designated for the approach. A shifting of the approach path by $5 \mathrm{~km}$, as indicated in Fig. 5, will improve the geometry to a extent that an averaging time $T_{\mathrm{av}}=20 \mathrm{~s}$ should guarantee the effectiveness of EA for the two DME stations designated for the approach.

\section{Conclusion And Outlook}

In this paper, we presented a method to analyze the effectiveness of EA for the geometry found in terrestrial radionavigation systems. We showed that EA is least effective if the aircraft is flying directly toward or away from the ground station. In that case, the zone in which a reflector not mitigable by means of EA can lie, is very big. Nevertheless, if the aircraft is traveling on a higher offset angle, the effectiveness of EA can be guaranteed if a small area around the transmitter is left clear of reflectors.

The presented method also allows the analysis of existing flight approaches. Current approaches are designed to fly directly toward terrestrial radionavigation aids, which reduces the effectiveness 


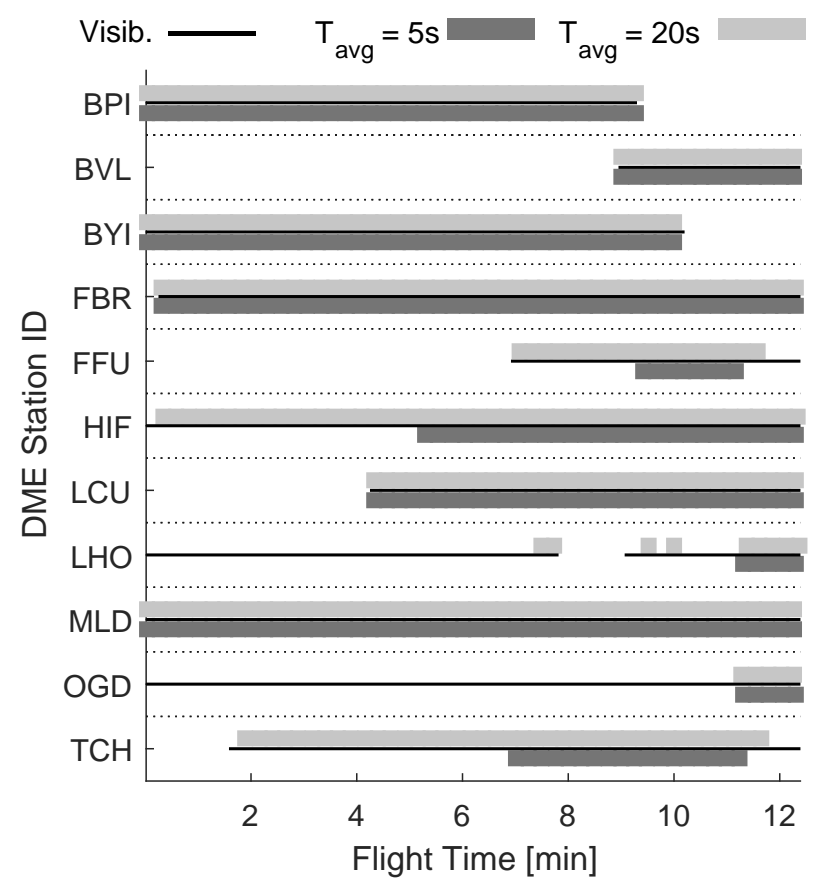

Fig. 5. The two thick lines show if the current geometry allows EA. The thin line indicates if a station is visible from the aircraft.

of EA. The presented method enables analysis of the effectiveness EA for multipath mitigation for selected ground station signals.

\section{REFERENCES}

[1] L. Eldredge, P. Enge, M. Harrison, R. Kenagy, S. Lo, R. Loh, R. Lilley, M. Narins, and R. Niles, "Alternative Positioning, Navigation and Timing (PNT) Study," in International Civil Aviation Organisation Navigation Systems Panel (NSP), (Montreal, Canada), 2010.

[2] M. Schnell, U. Epple, D. Shutin, and N. Schneckenburger, "LDACS: Future Aeronautical Communications for Air-Traffic Management," IEEE Commun. Mag., vol. 52, no. 5, pp. 104-110, 2014.

[3] R. Lilley and R. Erikson, "DME / DME for Alternate Position, Navigation, and Timing (APNT)," in FAA APNT White Paper, 2012.

[4] S. Lo, Y.-H. Chen, S. Zhang, and P. Enge, "Hybrid APNT : Terrestrial Radionavigation to Support Future Aviation Needs," in ION GNSS+, (Tampa (FL), USA), 2014.

[5] S. Lo, Y.-H. Chen, B. Segal, B. Peterson, P. Enge, R. Erikson, and R. Lilley, "Containing a difficult target: Techniques for mitigating DME multipath to Alternative Position Navigation and Timing (APNT)," in ION ITM, (San Diego (CA), USA), 2014.

[6] K. Li and W. Pelgrum, "Flight Test Evaluation of Enhanced DME(eDME) Performance Enhancements," in ION GNSS+, (Nashville (TN), USA), 2012.

[7] N. Schneckenburger, D. Shutin, T. Jost, M. Walter, T. Thiasiriphet, A. Filip, and M. Schnell, "From L-Band Measurements to a Preliminary Channel Model for APNT," in ION GNSS+, (Tampa (FL), USA), 2014. 
[8] W. Pelgrum and K. Li, "An Investigation on the Contributing Factors of Enhanced DME Ranging Errors," in ION GNSS+, (Tampa (FL), USA), 2015.

[9] W. Beyer, CRC Standard Mathematical Tables and Formulae. Boca Raton, FL: CRC Press, 28 ed., 1987. 